\title{
The Contribution of Neurocognitive Situation, Physical Capacity and Daily Life Activities to Quality of Life after Childhood Acute Lymphoblastic Leukemia
}

Elif KELES ${ }^{1}$, Yagmur Çam ${ }^{2}$ Bülent Elbasan ${ }^{2}$, Azime Şebnem Soysal Acar ${ }^{1}$, Zühre Kaya ${ }^{1}$, İdil Yenicesu ${ }^{3}$, and Ulker Kocak ${ }^{4}$

${ }^{1}$ Gazi University Faculty of Medicine

${ }^{2}$ Gazi University

${ }^{3}$ Gazi University Medical Faculty

${ }^{4}$ Gazi University, Faculty of Medicine

July 20, 2020

\begin{abstract}
Background There are no extensive studies on the quality of life of (QL) in children who completed ALL treatment and currently living without any disease in Turkey. Our study aimed to analyze both the QL and the effects of physical, neurocognitive capacities on QL of children aged between 7-12 years who received treatment for ALL. Methods Pediatric Quality of Life Scale (PedsQL) cancer module 3.0 child and proxy report, for ages 5-7 and 8-12 years, WeeFIM scale, Bruininks-Oseretsky motor competency test short form (BOT-2), Raven progressive test matrices(RPM), reading, writing and mathematics assessment tools and sociodemographic information form were carried out to obtain information from the children and their family. Results There was no effect of the months since the remission on pain, anxiety, cognitive problems, perceived physical appearance, and the total QL scores of children and proxy reports $(\mathrm{p}>0.05)$. The physical capacities of children were significantly worse than healthy controls and have not reached the level of healthy children even after a long time since remission. There was a significant association between physical capacity and daily independent living status $(\mathrm{p}<0.001)$. Reading, writing and mathematical skills were significantly associated with the duration of remission $(\mathrm{p}<0.001)$ and the total score of Raven and PedsQL of those with mathematical difficulties were significantly lower than those without any $\operatorname{difficulty}(\mathrm{p}<0.05)$.
\end{abstract}

The Contribution of Neurocognitive Situation, Physical Capacity and Daily Life Activities to Quality of Life after Childhood Acute Lymphoblastic Leukemia

Elif Keles ${ }^{1}$, Yağmur Çam² ${ }^{2}$ Bulent Elbasan ${ }^{2}$, Şebnem Soysal Acar ${ }^{1}$, Zuhre Kaya ${ }^{3}$, İdil Yenicesu ${ }^{3}$, Ülker $\mathrm{Koçak}^{3}$

${ }^{1}$ Gazi University Faculty of Medicine, Department of Pediatrics, Ankara,Turkey

${ }^{2}$ Gazi University Faculty of Health Sciences, Physiotherapy and Rehabilitation, Ankara, Turkey

${ }^{3}$ Gazi University Faculty of Medicine, Department of Pediatrics, Division of Pediatric Hematology,Ankara, Turkey

Abbrevations

\begin{tabular}{ll}
\hline ALL & Acute Lymphoblastic Leukemia \\
\hline B ALL & B cell Acute Lymphoblastic Leukemia
\end{tabular}




\begin{tabular}{ll}
\hline ALL & Acute Lymphoblastic Leukemia \\
\hline T ALL & T cell Acute Lymphoblastic Leukemia \\
QL & Quality of Life \\
PedsQL & Pediatric Quality of Life Scale \\
WeeFIM & The Functional Independence Measure (WeeFIM) \\
BOT-2 & Bruininks-Oseretsky Motor Competency Test Short Form \\
RPM & Raven Progressive Test Matrices \\
CNS & Central Nervous System \\
ALL-BFM 2000 & Acute Lymphoblastic Leukemia Berlin Frankfurt Münster 2000 Chemotherapy Protocol \\
\hline
\end{tabular}

\section{Corresponding author:}

Elif Keles, Department of Pediatrics, Gazi University Faculty of Medicine, Emniyet Mahallesi, Mevlana Bulvarı, Number: 29, 06560, Yenimahalle, Ankara, Turkey

Phone: +90 536 6741270; e-mail address: elifkeles.dr@gmail.com

\section{Grant Support \& Financial Disclosures}

None

\section{Acknowledgments}

The authors would like to thank all the children and parents who participated in the study. They acknowledge the support of Dilek EKİĊ, PhD, Professor, Nursing Management Department, Gazi University Faculty of Health Science and her valuable input on the statistical analysis of this study

\section{Authorship Contributions}

Concept: Elif Keleş, Ülker Koçak

Design: Elif Keleş, Bulent Elbasan, Şebnem Soysal Acar ,Ülker Koçak

Data Collection or Processing: Elif Keleş, Yağmur Çam, Bulent Elbasan, Şebnem Soysal Acar , Zuhre Kaya, İdil Yenicesu

Analysis or Interpretation: Elif Keleş, Yağmur Çam, Bulent Elbasan, Şebnem Soysal Acar, Ülker Koçak

Literature Search: Elif Keleş, Ülker Koçak, Zuhre Kaya, İdil Yenicesu ,

Writing: Elif Keleş, Ülker Koçak

Conflict of Interest: The authors of this paper have no conflicts of interest, including specific financial interests, relationships, and/or affiliations relevant to the subject matter or materials included.

Abstract word count: 199

The text word count : 3303

Keywords: ALL, Quality of Life, Neurocognitive and Physical Outcome,

The number of table : 3

\section{ABSTRACT}

\section{Background}

There are no extensive studies on the QL in children who completed ALL treatment and currently living without any disease in Turkey. Our study aimed to analyze both the QL and the effects of physical, neurocognitive capacities on QL of children aged between 7-12 years who received treatment for ALL. 


\section{Methods}

PedsQL cancer module 3.0 child and proxy report, for ages 5-7 and 8-12 years, WeeFIM scale, BOT-2, RPM, reading, writing and mathematics assessment tools and sociodemographic information form were carried out to obtain information from the children and their family.

\section{Results}

There was no effect of the months since the remission on pain, anxiety, cognitive problems, perceived physical appearance, and the total QL scores of children and proxy reports $(\mathrm{p}>0.05)$.

The physical capacities of children were significantly worse than healthy controls and have not reached the level of healthy children even after a long time since remission. There was a significant association between physical capacity and daily independent living status $(\mathrm{p}<0.001)$.

Reading, writing and mathematical skills were significantly associated with the duration of remission $(\mathrm{p}<0.001)$ and the total score of RPM and PedsQL of those with mathematical difficulties were significantly lower than those without any $\operatorname{difficulty}(\mathrm{p}<0.05)$.

\section{Hosted file}

manuscript1907.docx available at https://authorea.com/users/344281/articles/470883-thecontribution-of-neurocognitive-situation-physical-capacity-and-daily-life-activities-toquality-of-life-after-childhood-acute-lymphoblastic-leukemia 